\title{
Optimal Energy Decay for a Transmission Problem of Waves Under a Nonlocal Boundary Control
}

\author{
Halim Atoui and Abbes Benaissa*
}

\begin{abstract}
In this paper, we consider a transmission problem in the presence of a boundary control condition of nonlocal type. We prove well-posedness by using the semigroup theory. Also we establish an optimal decay result by frequency domain method and Borichev-Tomilov theorem.
\end{abstract}

\section{Introduction}

In this paper we study a transmission wave system with boundary control of nonlocal type given by

$$
\begin{aligned}
\rho_{1} u_{t t}(x, t)-\tau_{1} u_{x x}(x, t)=0 & \text { in }\left(0, l_{0}\right) \times(0,+\infty), \\
\rho_{2} v_{t t}(x, t)-\tau_{2} v_{x x}(x, t)=0 & \text { in }\left(l_{0}, L\right) \times(0,+\infty),
\end{aligned}
$$

where $\rho_{1}, \rho_{2}, \tau_{1}$ and $\tau_{2}$ are positive constants that represent the densities and tensions of the strings $u$ and $v$, respectively, and the initial conditions are

$$
u(x, 0)=u_{0}(x), \quad u_{t}(x, 0)=u_{1}(x), \quad v(x, 0)=v_{0}(x), \quad v_{t}(x, 0)=v_{1}(x) .
$$

The transmission condition is

$$
u\left(l_{0}, t\right)=v\left(l_{0}, t\right), \quad \rho_{2} \tau_{1} u_{x}\left(l_{0}, t\right)=\rho_{1} \tau_{2} v_{x}\left(l_{0}, t\right), \quad \forall t \in(0,+\infty),
$$

followed by the boundary conditions

$$
u(0, t)=0, \quad \tau_{2} v_{x}(L, t)+\gamma \rho_{2} \partial_{t}^{\alpha, \eta} v(L, t)=0, \quad \forall t \in(0,+\infty)
$$

and conditions of compatibility

$$
u_{0}\left(l_{0}\right)=v_{0}\left(l_{0}\right), \quad u_{1}\left(l_{0}\right)=v_{1}\left(l_{0}\right), \quad \rho_{2} \tau_{1} u_{0 x}\left(l_{0}\right)=\rho_{1} \tau_{2} v_{0 x}\left(l_{0}\right),
$$

Received September 25, 2018; Accepted January 27, 2019.

Communicated by Cheng-Hsiung Hsu.

2010 Mathematics Subject Classification. 93D15, 35B40, 47D03, 74D05.

Key words and phrases. transmission problem of waves, boundary dissipation of nonlocal type, frequency domain method, optimal polynomial stability.

*Corresponding author. 
where $\gamma>0$, the initial data $\left(u_{0}, u_{1}, v_{0}, v_{1}\right)$ belong to a suitable function space. The notation $\partial_{t}^{\alpha, \eta}$ stands for the generalized Caputo's fractional derivative of order $\alpha, 0<\alpha<$ 1, with respect to the time variable (see Choi and MacCamy [7] and E. Blanc, G. Chiavassa, and B. Lombard $[5]$ ). It is defined as follows

$$
\partial_{t}^{\alpha, \eta} w(t)=\frac{1}{\Gamma(1-\alpha)} \int_{0}^{t}(t-s)^{-\alpha} e^{-\eta(t-s)} \frac{d w}{d s}(s) d s, \quad \eta \geq 0 .
$$

Very little attention has been paid to this type of feedback. Moreover, fractional derivatives involve singular and nonintegrable kernels $\left(t^{\alpha}, 0<\alpha<1\right)$. This leads to substantial mathematical difficulties such as numerical approximation.

In [13, B. Mbodje investigates the decay rate of the energy of the wave equation with a boundary nonlocal control, that is,

$$
\begin{array}{ll}
u_{t t}(x, t)-u_{x x}(x, t)=0 & \text { in }(0, L) \times(0,+\infty), \\
u(0, t)=0 & \text { on }(0,+\infty), \\
u_{x}(L, t)+\gamma \partial_{t}^{\alpha, \eta} u_{t}(L, t)=0 & \text { on }(0,+\infty), \\
u(x, 0)=u_{0}(x), \quad u_{t}(x, 0)=u_{1}(x) & \text { on }(0, L) .
\end{array}
$$

Using energy methods, he proves strong asymptotic stability under the condition $\eta=0$ and a polynomial type decay rate $E(t) \leq c / t$ if $\eta \neq 0$.

Very recently, in [1], Benaissa et al. considered the Euler-Bernoulli beam equation with boundary dissipation of nonlocal type defined by

$$
\begin{array}{ll}
u_{t t}(x, t)+u_{x x x x}(x, t)=0 & \text { in }(0, L) \times(0,+\infty), \\
u(0, t)=u_{x}(0, t)=0 & \text { on }(0,+\infty), \\
u_{x x}(L, t)=0 & \text { on }(0,+\infty), \\
u_{x x x}(L, t)-\gamma \partial_{t}^{\alpha, \eta} u_{t}(L, t)=0 & \text { on }(0,+\infty), \\
u(x, 0)=u_{0}(x), \quad u_{t}(x, 0)=u_{1}(x) & \text { on }(0, L) .
\end{array}
$$

They proved, under the condition $\eta=0$, by a spectral analysis, the non uniform stability. On the other hand, for $\eta>0$, they also proved that the energy of system (1.6) decays as time goes to infinity as $t^{-1 /(1-\alpha)}$.

The question we are interested in this paper is what are the stability properties of our system (1.1)-1.5. Indeed, this system involves two wave equations coupled at interface with only one nonlocal control acting on a part of the boundary of the second equation. So, from the mathematical point of view, it is important to study the stability of an equation of 1D waves with discontinuous coefficients in a bounded domain. Moreover, this system happens frequently in applications where the domain is occupied by two different types of materials, that is, while one of them is simply elastic, the other is subject to the 
action of an external force. Let us mention here that the case $\alpha=1$ corresponds to a static boundary control, that is,

$$
\tau_{2} v_{x}(L, t)+\gamma \rho_{2} v_{t}(L, t)=0, \quad \forall t \in(0,+\infty)
$$

It is well known that the energy of the solution decays exponentially under the conditions (see 17$])$

$$
\chi=\frac{\tau_{1}}{\rho_{1}}-\frac{\tau_{2}}{\rho_{2}}>0
$$

Nowadays, fractional calculus is not only important from the theoretical point of view but also for applications. The main reason for the diffusion of fractional calculus is that it actually provides a more accurate tool to describe several physical systems. For instance, phenomena such as heat conduction through a semi-infinite solid, water flowing through a porous dyke or infinite lossy transmission lines are indeed fractional. In many industrial and research fields, fractional calculus can be conveniently used. Among these, relevant research topics are electrical circuits, chemical processes, signal processing, viscoelasticity, chaos theory, and obviously control systems (see $3,4,12,15,18,19$ ). In our case, the fractional dissipations may simply describe an active boundary viscoelastic damper designed for the purpose of reducing the vibrations (see [13, 14]).

The organization of this paper is as follows. In Section 2, first we show that the system (1.1) can be replaced by an augmented model by coupling the transmission wave system with a suitable diffusion equation that can be reformulate into classical input output dynamic systems and we deduce the well-posedness property of the problem by the semigroup approach. Secondly, using a criteria of Arendt-Batty [2] we show that the augmented model is strongly stable in the absence of compactness of the resolvent. In Section 3, we show the lack of exponential stability by spectral analysis. In Section 4. we show an optimal energy decay rate depending on the parameter $\alpha$. The proof heavily relies on a precise estimate of the resolvent of the generator associated to the semi-group and Borichev-Tomilov theorem.

\section{Well-posedness and strong stability}

This section is concerned with the reformulation of the model (1.1) into an augmented system. For that, we need the following claims.

Theorem 2.1. (see [13]) Let $\mu$ be the function

$$
\mu(\xi)=|\xi|^{(2 \alpha-1) / 2}, \quad-\infty<\xi<+\infty, 0<\alpha<1 .
$$


Then the relationship between the 'input' $U$ and the 'output' $O$ of the system

$$
\begin{gathered}
\partial_{t} \phi(\xi, t)+\left(\xi^{2}+\eta\right) \phi(\xi, t)-U(t) \mu(\xi)=0, \quad-\infty<\xi<+\infty, \eta \geq 0, t>0, \\
\phi(\xi, 0)=0 \\
O(t)=(\pi)^{-1} \sin (\alpha \pi) \int_{-\infty}^{+\infty} \mu(\xi) \phi(\xi, t) d \xi
\end{gathered}
$$

is given by

$$
O=I^{1-\alpha, \eta} U
$$

where

$$
\left[I^{\alpha, \eta} f\right](t)=\frac{1}{\Gamma(\alpha)} \int_{0}^{t}(t-\tau)^{\alpha-1} e^{-\eta(t-\tau)} f(\tau) d \tau
$$

Lemma 2.2. (see [1]) If $\left.\left.\lambda \in D_{\eta}=\mathbb{C} \backslash\right]-\infty,-\eta\right]$ then

$$
F(\lambda)=\int_{-\infty}^{+\infty} \frac{\mu^{2}(\xi)}{\lambda+\eta+\xi^{2}} d \xi=\frac{\pi}{\sin \alpha \pi}(\lambda+\eta)^{\alpha-1}
$$

\subsection{Well-posedness}

We are now in a position to reformulate system (1.1). Indeed, by using Theorem 2.1. system (1.1) becomes

$$
\begin{array}{ll}
\rho_{1} u_{t t}(x, t)-\tau_{1} u_{x x}(x, t)=0 & \text { in }\left(0, l_{0}\right) \times(0,+\infty), \\
\rho_{2} v_{t t}(x, t)-\tau_{2} v_{x x}(x, t)=0 & \text { in }\left(l_{0}, L\right) \times(0,+\infty), \\
\partial_{t} \phi(\xi, t)+\left(\xi^{2}+\eta\right) \phi(\xi, t)-v_{t}(L, t) \mu(\xi)=0 & \text { in }(-\infty, \infty) \times(0,+\infty), \\
u\left(l_{0}, t\right)=v\left(l_{0}, t\right), \quad \rho_{2} \tau_{1} u_{x}\left(l_{0}, t\right)=\rho_{1} \tau_{2} v_{x}\left(l_{0}, t\right) & \text { on }(0,+\infty), \\
u(0, t)=0 & \text { on }(0,+\infty), \\
\tau_{2} v_{x}(L, t)+\zeta \rho_{2} \int_{-\infty}^{+\infty} \mu(\xi) \phi(\xi, t) d \xi=0 & \text { on }(0,+\infty), \\
u(x, 0)=u_{0}(x), \quad u_{t}(x, 0)=u_{1}(x) & \text { on }\left(0, l_{0}\right), \\
v(x, 0)=v_{0}(x), \quad v_{t}(x, 0)=v_{1}(x) & \text { on }\left(l_{0}, L\right),
\end{array}
$$

where $\zeta=(\pi)^{-1} \sin (\alpha \pi) \gamma$. For a solution $(u, v, \phi)$ of 2.1 , we define the energy

$$
\begin{aligned}
E(t)= & \frac{1}{2} \int_{0}^{l_{0}}\left(\left|u_{t}\right|^{2}+\frac{\tau_{1}}{\rho_{1}}\left|u_{x}\right|^{2}\right) d x+\frac{1}{2} \int_{l_{0}}^{L}\left(\left|v_{t}\right|^{2}+\frac{\tau_{2}}{\rho_{2}}\left|v_{x}\right|^{2}\right) d x \\
& +\frac{\zeta}{2} \int_{-\infty}^{+\infty}|\phi(\xi, t)|^{2} d \xi .
\end{aligned}
$$

Lemma 2.3. Let $(u, v, \phi)$ be a regular solution of the problem (2.1). Then, the energy functional defined by 2.2 satisfies

$$
E^{\prime}(t)=-\zeta \int_{-\infty}^{+\infty}\left(\xi^{2}+\eta\right)|\phi(\xi, t)|^{2} d \xi \leq 0
$$


Proof. Multiplying the first equation in (2.1) by $\bar{u}_{t}$, integrating by parts over $\left(0, l_{0}\right)$, we obtain

$$
\frac{1}{2} \frac{d}{d t} \int_{0}^{l_{0}}\left(\left|u_{t}\right|^{2}+\frac{\tau_{1}}{\rho_{1}}\left|u_{x}\right|^{2}\right) d x-\frac{\tau_{1}}{\rho_{1}} \Re u_{x}\left(l_{0}\right) \bar{u}_{t}\left(l_{0}\right)=0 .
$$

Multiplying the second equation in 2.1 by $\bar{v}_{t}$, integrating by parts over $\left(l_{0}, L\right)$, we obtain

$$
\frac{1}{2} \frac{d}{d t} \int_{0}^{l_{0}}\left(\left|v_{t}\right|^{2}+\frac{\tau_{2}}{\rho_{2}}\left|v_{x}\right|^{2}\right) d x+\frac{\tau_{2}}{\rho_{2}} \Re v_{x}\left(l_{0}\right) \bar{v}_{t}\left(l_{0}\right)-\frac{\tau_{2}}{\rho_{2}} \Re v_{x}(L) \bar{v}_{t}(L, t)=0 .
$$

Adding the two equations above, we obtain

$$
\frac{1}{2} \frac{d}{d t} \int_{0}^{l_{0}}\left(\left|u_{t}\right|^{2}+\frac{\tau_{1}}{\rho_{1}}\left|u_{x}\right|^{2}\right) d x+\frac{1}{2} \frac{d}{d t} \int_{0}^{l_{0}}\left(\left|v_{t}\right|^{2}+\frac{\tau_{2}}{\rho_{2}}\left|v_{x}\right|^{2}\right) d x-\frac{\tau_{2}}{\rho_{2}} \Re v_{x}(L) \bar{v}_{t}(L, t)=0 .
$$

From the boundary condition (the sixth equation in (2.1)), we have

$$
\begin{aligned}
& \frac{1}{2} \frac{d}{d t}\left[\int_{0}^{l_{0}}\left(\left|u_{t}\right|^{2}+\frac{\tau_{1}}{\rho_{1}}\left|u_{x}\right|^{2}\right) d x+\int_{0}^{l_{0}}\left(\left|v_{t}\right|^{2}+\frac{\tau_{2}}{\rho_{2}}\left|v_{x}\right|^{2}\right) d x\right] \\
+ & \zeta \bar{v}_{t}(L, t) \int_{-\infty}^{+\infty} \mu(\xi) \phi(\xi, t) d \xi=0 .
\end{aligned}
$$

Multiplying the third equation in 2.1$)$ by $\zeta \bar{\phi}_{t}$ and integrating over $(-\infty,+\infty)$, to obtain

$$
\frac{\zeta}{2} \frac{d}{d t}\|\phi\|_{2}^{2}+\zeta \int_{-\infty}^{+\infty}\left(\xi^{2}+\eta\right)|\phi(\xi, t)|^{2} d \xi-\zeta \Re v_{t}(L, t) \int_{-\infty}^{+\infty} \mu(\xi) \bar{\phi}(\xi, t) d \xi=0
$$

Consequently, it is resulted from $2.2,2.3$ and 2.4 that

$$
E^{\prime}(t)=-\zeta \int_{-\infty}^{+\infty}\left(\xi^{2}+\eta\right)|\phi(\xi, t)|^{2} d \xi
$$

This completes the proof of the lemma.

We now discuss the well-posedness of 2.1). For this purpose, we introduce the following space

$$
H_{*}^{1}\left(0, l_{0}\right)=\left\{u \in H^{1}\left(0, l_{0}\right): u(0)=0\right\} .
$$

We then reformulate (2.1) into a semigroup setting. Let $\widetilde{u}=u_{t}, \widetilde{v}=v_{t}$, and set

$$
\mathcal{H}=\left\{H_{*}^{1}\left(0, l_{0}\right) \times L^{2}\left(0, l_{0}\right) \times H^{1}\left(l_{0}, L\right) \times L^{2}\left(l_{0}, L\right) \times L^{2}(-\infty,+\infty) \backslash u\left(l_{0}\right)=v\left(l_{0}\right)\right\}
$$

equipped with the inner product

$$
\left\langle U, U_{1}\right\rangle_{\mathcal{H}}=\int_{0}^{l_{0}}\left(\widetilde{u} \overline{\widetilde{u}}_{1}+\frac{\tau_{1}}{\rho_{1}} u_{x} \bar{u}_{1 x}\right) d x+\int_{l_{0}}^{L}\left(\widetilde{v} \overline{\widetilde{v}}_{1}+\frac{\tau_{2}}{\rho_{2}} v_{x} \bar{v}_{1 x}\right) d x+\zeta \int_{-\infty}^{+\infty} \phi \bar{\phi}_{1} d \xi
$$

for any $U=(u, \widetilde{u}, v, \widetilde{v}, \phi)^{T}$ and $U_{1}=\left(u_{1}, \widetilde{u}_{1}, v_{1}, \widetilde{v}_{1}, \phi_{1}\right)^{T}$. 
Let $U=(u, \widetilde{u}, v, \widetilde{v}, \phi)^{T}$ and rewrite 2.1 as

$$
U^{\prime}=\mathcal{A} U, \quad U(0)=U_{0}=\left(u_{0}, u_{1}, v_{0}, v_{1}, \phi_{0}\right),
$$

where the operator $\mathcal{A}$ is defined by

$$
\mathcal{A}\left(\begin{array}{c}
u \\
\widetilde{u} \\
v \\
\widetilde{v} \\
\phi
\end{array}\right)=\left(\begin{array}{c}
\widetilde{u} \\
\frac{\tau_{1}}{\rho_{1}} u_{x x} \\
\widetilde{v} \\
\frac{\tau_{2}}{\rho_{2}} v_{x x} \\
-\left(\xi^{2}+\eta\right) \phi+\widetilde{v}(L) \mu(\xi)
\end{array}\right)
$$

The domain of $\mathcal{A}$ is

$$
D(\mathcal{A})=\left\{\begin{array}{c}
(u, \widetilde{u}, v, \widetilde{v}, \phi)^{T} \text { in } \mathcal{H}: u \in H^{2}(0, L) \cap H_{*}^{1}\left(0, l_{0}\right), \widetilde{u} \in H_{*}^{1}\left(0, l_{0}\right), \\
v \in H^{2}\left(l_{0}, L\right), \widetilde{v} \in H^{1}\left(l_{0}, L\right), u\left(l_{0}\right)=v\left(l_{0}\right), \rho_{2} \tau_{1} u_{x}\left(l_{0}\right)=\rho_{1} \tau_{2} v_{x}\left(l_{0}\right), \\
\widetilde{u}\left(l_{0}\right)=\widetilde{v}\left(l_{0}\right),-\left(\xi^{2}+\eta\right) \phi+\widetilde{v}(L) \mu(\xi) \in L^{2}(-\infty,+\infty), \\
\tau_{2} v_{x}(L)+\zeta \rho_{2} \int_{-\infty}^{+\infty} \mu(\xi) \phi(\xi) d \xi=0, \\
|\xi| \phi \in L^{2}(-\infty,+\infty)
\end{array}\right\} .
$$

The well-posedness of problem 2.1 is ensured by the following theorem.

Theorem 2.4 (Existence and uniqueness). (1) If $U_{0} \in D(\mathcal{A})$, then system (2.5) has a unique strong solution

$$
U \in C^{0}\left(\mathbb{R}_{+}, D(\mathcal{A})\right) \cap C^{1}\left(\mathbb{R}_{+}, \mathcal{H}\right) .
$$

(2) If $U_{0} \in \mathcal{H}$, then system 2.5 has a unique weak solution

$$
U \in C^{0}\left(\mathbb{R}_{+}, \mathcal{H}\right)
$$

Proof. We show that $\mathcal{A}$ is monotone maximal. First, it is easy to see that

$$
\Re\langle\mathcal{A} U, U\rangle_{\mathcal{H}}=-E^{\prime}(t)=-\zeta \int_{-\infty}^{+\infty}\left(\xi^{2}+\eta\right)|\phi(\xi)|^{2} d \xi
$$

For the maximality, let $F=\left(f_{1}, f_{2}, f_{3}, f_{4}, f_{5}\right)^{T} \in \mathcal{H}$ and look for $U=(u, \widetilde{u}, v, \widetilde{v}, \phi)^{T} \in$ $D(\mathcal{A})$ satisfying $\lambda U-\mathcal{A} U=F$ for $\lambda>0$, that is,

$$
\begin{gathered}
\lambda u-\widetilde{u}=f_{1}, \quad \lambda \widetilde{u}-\frac{\tau_{1}}{\rho_{1}} u_{x x}=f_{2}, \quad \lambda v-\widetilde{v}=f_{3}, \\
\lambda \widetilde{v}-\frac{\tau_{2}}{\rho_{2}} v_{x x}=f_{4}, \quad \lambda \phi+\left(\xi^{2}+\eta\right) \phi-\widetilde{v}(L) \mu(\xi)=f_{5} .
\end{gathered}
$$


Assume that with the suitable regularity we have found $u$ and $v$, then

$$
\widetilde{u}=\lambda u-f_{1}, \quad \widetilde{v}=\lambda v-f_{3} .
$$

It is clear that $\widetilde{u} \in H_{*}^{1}\left(0, l_{0}\right)$ and $\widetilde{v} \in H^{1}\left(l_{0}, L\right)$. Furthermore, by $(2.9)$ we can find $\phi$ as

$$
\phi=\frac{f_{5}(\xi)+\mu(\xi) \widetilde{v}(L)}{\xi^{2}+\eta+\lambda} .
$$

From $(2.9)$ and $(2.10)$ one can see that the functions $u$ and $v$ satisfy the following system

$$
\lambda^{2} u-\frac{\tau_{1}}{\rho_{1}} u_{x x}=f_{2}+\lambda f_{1}, \quad \lambda^{2} v-\frac{\tau_{2}}{\rho_{2}} v_{x x}=f_{4}+\lambda f_{3} .
$$

Solving system (2.12) is equivalent to finding $u \in H^{2} \cap H_{*}^{1}\left(0, l_{0}\right)$ and $v \in H^{2}\left(l_{0}, L\right)$ such that

$$
\begin{aligned}
\int_{0}^{l_{0}}\left(\lambda^{2} u \bar{w}-\frac{\tau_{1}}{\rho_{1}} u_{x x} \bar{w}\right) d x & =\int_{0}^{l_{0}}\left(f_{2}+\lambda f_{1}\right) \bar{w} d x \\
\int_{l_{0}}^{L}\left(\lambda^{2} v \bar{\chi}-\frac{\tau_{2}}{\rho_{2}} v_{x x} \bar{\chi}\right) d x & =\int_{l_{0}}^{L}\left(f_{4}+\lambda f_{3}\right) \bar{\chi} d x
\end{aligned}
$$

for all $w \in H_{*}^{1}\left(0, l_{0}\right)$ and $\chi \in H^{1}\left(l_{0}, L\right)$. From (2.13) and 2.11) one can see that the functions $u$ and $v$ satisfy the following system

$$
\begin{aligned}
& \int_{0}^{l_{0}}\left(\lambda^{2} u \bar{w}+\frac{\tau_{1}}{\rho_{1}} u_{x} \bar{w}_{x}\right) d x+\int_{l_{0}}^{L}\left(\lambda^{2} v \bar{\chi}+\frac{\tau_{2}}{\rho_{2}} v_{x} \bar{\chi}_{x}\right) d x+\widetilde{\zeta} \lambda v(L) \bar{\chi}(L) \\
= & \int_{0}^{l_{0}}\left(f_{2}+\lambda f_{1}\right) \bar{w} d x+\int_{l_{0}}^{L}\left(f_{4}+\lambda f_{3}\right) \bar{\chi} d x \\
& -\zeta \int_{-\infty}^{+\infty} \frac{\mu(\xi)}{\xi^{2}+\eta+\lambda} f_{5}(\xi) d \xi \bar{\chi}(L)+\widetilde{\zeta} f_{3}(L) \bar{\chi}(L),
\end{aligned}
$$

where $\widetilde{\zeta}=\zeta \int_{-\infty}^{+\infty} \frac{\mu^{2}(\xi)}{\xi^{2}+\eta+\lambda} d \xi$. Consequently, problem 2.14 is equivalent to the problem

$$
a((u, v),(w, \chi))=L(w, \chi),
$$

where the bilinear form $a:\left[H_{*}^{1}\left(0, l_{0}\right) \times H^{1}\left(l_{0}, L\right)\right]^{2} \rightarrow \mathbb{R}$ and the linear form $L: H_{*}^{1}\left(0, l_{0}\right) \times$ $H^{1}\left(l_{0}, L\right) \rightarrow \mathbb{R}$ are defined by

$$
a((u, v),(w, \chi))=\int_{0}^{l_{0}}\left(\lambda^{2} u \bar{w}+\frac{\tau_{1}}{\rho_{1}} u_{x} \bar{w}_{x}\right) d x+\int_{l_{0}}^{L}\left(\lambda^{2} v \bar{\chi}+\frac{\tau_{2}}{\rho_{2}} v_{x} \bar{\chi}_{x}\right) d x+\widetilde{\zeta} \lambda v(L) \bar{\chi}(L)
$$

and

$$
\begin{aligned}
L(w, \chi)= & \int_{0}^{l_{0}}\left(f_{2}+\lambda f_{1}\right) \bar{w} d x+\int_{l_{0}}^{L}\left(f_{4}+\lambda f_{3}\right) \bar{\chi} d x \\
& -\zeta \int_{-\infty}^{+\infty} \frac{\mu(\xi)}{\xi^{2}+\eta+\lambda} f_{5}(\xi) d \xi \bar{\chi}(L)+\widetilde{\zeta} f_{3}(L) \bar{\chi}(L) .
\end{aligned}
$$


It is easy to verify that $a$ is continuous and coercive, and $L$ is continuous. Applying the Lax-Milgram theorem, we infer that for all $(w, \chi) \in H_{*}^{1}\left(0, l_{0}\right) \times H^{1}\left(l_{0}, L\right)$ problem (2.15) has a unique solution $(u, v) \in H_{*}^{1}\left(0, l_{0}\right) \times H^{1}\left(l_{0}, L\right)$. Applying the classical elliptic regularity, it follows from (2.14) that $(u, v) \in H^{2}\left(0, l_{0}\right) \times H^{2}\left(l_{0}, L\right)$. Therefore, the operator $\lambda I-\mathcal{A}$ is surjective for any $\lambda>0$. At last, the result of Theorem 2.4 follows from the Hille-Yosida theorem.

\subsection{Strong stability of the system}

Because of the unboundedness of the $\xi$-domain for the diffusive equation, the resolvent of $\mathcal{A}$ is not compact, then the classical methods such as LaSalle's invariance principle or the spectrum decomposition theory of Benchimol are not applicable in this case. We use a general criteria of Arendt-Batty (see [2,11]), following which a $C_{0}$-semigroup of contractions $e^{t \mathcal{A}}$ in a Banach space is strongly stable, if $\mathcal{A}$ has no pure imaginary eigenvalues and $\sigma(\mathcal{A}) \cap i \mathbb{R}$ contains only a countable number of elements. Our main result is the following theorem.

Theorem 2.5. The $C_{0}$-semigroup $e^{t \mathcal{A}}$ is strongly stable in $\mathcal{H}$; i.e., for all $U_{0} \in \mathcal{H}$, the solution of (2.5) satisfies

$$
\lim _{t \rightarrow \infty}\left\|e^{t \mathcal{A}} U_{0}\right\|_{\mathcal{H}}=0 .
$$

For the proof of Theorem 2.5, we need the following two lemmas.

Lemma 2.6. $\mathcal{A}$ does not have eigenvalues on $i \mathbb{R}$.

Proof. We make a distinction between $i \lambda=0$ and $i \lambda \neq 0$.

Step 1. Solving for $\mathcal{A} U=0$ leads to $U=0$, thanks to the boundary conditions in 2.7). Hence, $i \lambda=0$ is not an eigenvalue of $\mathcal{A}$.

Step 2. We will argue by contradiction. Let us suppose that $\lambda \in \mathbb{R}, \lambda \neq 0$ and $U \neq 0$ such that $\mathcal{A} U=i \lambda U$. Then, we get

$$
\begin{gathered}
i \lambda u-\widetilde{u}=0, \\
i \lambda \widetilde{u}-\frac{\tau_{1}}{\rho_{1}} u_{x x}=0, \\
i \lambda v-\widetilde{v}=0, \\
i \lambda \widetilde{v}-\frac{\tau_{2}}{\rho_{2}} v_{x x}=0, \\
i \lambda \phi+\left(\xi^{2}+\eta\right) \phi-\widetilde{v}(L) \mu(\xi)=0 .
\end{gathered}
$$

Then, from 2.8$)$ we have

$$
\phi \equiv 0
$$


From 2.16e, we have

$$
\widetilde{v}(L)=0
$$

Hence, from 2.16c and the relation $\tau_{2} v_{x}(L)+\zeta \rho_{2} \int_{-\infty}^{+\infty} \mu(\xi) \phi(\xi) d \xi=0$, we obtain

$$
v(L)=0 \quad \text { and } \quad v_{x}(L)=0 .
$$

Inserting 2.16c into 2.16d, we get

$$
-\lambda^{2} v-\frac{\tau_{2}}{\rho_{2}} v_{x x}=0
$$

The solution of the equation $(2.18)$ is given by

$$
v(x)=c_{1} \cos \frac{\lambda}{r_{2}} x+c_{2} \sin \frac{\lambda}{r_{2}} x, \quad r_{2}=\sqrt{\frac{\tau_{2}}{\rho_{2}}} .
$$

From boundary conditions 2.17 , we deduce that

$$
v \equiv 0 .
$$

Now, from the boundary transmission conditions, we get

$$
u\left(l_{0}\right)=u_{x}\left(l_{0}\right)=0 .
$$

Similarly, we deduce that

$$
u \equiv 0 .
$$

Therefore $U=0$. Consequently, $\mathcal{A}$ does not have purely imaginary eigenvalues.

Lemma 2.7. If $\lambda \neq 0$, the operator $i \lambda I-\mathcal{A}$ is surjective. If $\lambda=0$ and $\eta \neq 0$, the operator $i \lambda I-\mathcal{A}$ is surjective.

Proof. Case 1: $\lambda \neq 0$. Let $F=\left(f_{1}, f_{2}, f_{3}, f_{4}, f_{5}\right)^{T} \in \mathcal{H}$ be given and let $X=(u, \widetilde{u}, v, \widetilde{v}, \phi)^{T}$ $\in D(\mathcal{A})$ be such that

$$
(i \lambda I-\mathcal{A}) X=F
$$

Equivalently, we have

$$
\begin{gathered}
i \lambda u-\widetilde{u}=f_{1}, \\
i \lambda \widetilde{u}-\frac{\tau_{1}}{\rho_{1}} u_{x x}=f_{2}, \\
i \lambda v-\widetilde{v}=f_{3}, \\
i \lambda \widetilde{v}-\frac{\tau_{2}}{\rho_{2}} v_{x x}=f_{4}, \\
i \lambda \phi+\left(\xi^{2}+\eta\right) \phi-\widetilde{v}(L) \mu(\xi)=f_{5} .
\end{gathered}
$$


Inserting 2.19a , 2.19c into 2.19b and $2.19 \mathrm{~d})$, we get

$$
-\lambda^{2} u-r_{1} u_{x x}=\left(f_{2}+i \lambda f_{1}\right), \quad-\lambda^{2} v-r_{2} v_{x x}=\left(f_{4}+i \lambda f_{3}\right) .
$$

Solving system 2.20$)$ is equivalent to finding $(u, v) \in H^{2} \cap H_{*}^{1}\left(0, l_{0}\right) \times H^{2}\left(l_{0}, L\right)$ such that

$$
\begin{aligned}
\int_{0}^{l_{0}}\left(-\lambda^{2} u \bar{w}-r_{1} u_{x x} \bar{w}\right) d x & =\int_{0}^{l_{0}}\left(f_{2}+i \lambda f_{1}\right) \bar{w} d x \\
\int_{l_{0}}^{L}\left(-\lambda^{2} v \bar{\chi}-r_{2} v_{x x} \bar{\chi}\right) d x & =\int_{l_{0}}^{L}\left(f_{4}+i \lambda f_{3}\right) \bar{\chi} d x
\end{aligned}
$$

for all $(w, \chi) \in H_{*}^{1}\left(0, l_{0}\right) \times H^{1}\left(l_{0}, L\right)$. By using $\left.2.19 \mathrm{c}\right)$ and $2.19 \mathrm{e}$ the functions $u$ and $v$ satisfying the following system

$$
\begin{aligned}
& \int_{0}^{l_{0}}\left(-\lambda^{2} u \bar{w}+\frac{\tau_{1}}{\rho_{1}} u_{x} \bar{w}_{x}\right) d x+\int_{l_{0}}^{L}\left(-\lambda^{2} v \bar{\chi}+\frac{\tau_{2}}{\rho_{2}} v_{x} \bar{\chi}_{x}\right) d x+\widetilde{\zeta} i \lambda v(L) \bar{\chi}(L) \\
= & \int_{0}^{l_{0}}\left(f_{2}+i \lambda f_{1}\right) \bar{w} d x+\int_{l_{0}}^{L}\left(f_{4}+i \lambda f_{3}\right) \bar{\chi} d x \\
& -\zeta \int_{-\infty}^{+\infty} \frac{\mu(\xi)}{\xi^{2}+\eta+i \lambda} f_{5}(\xi) d \xi \bar{\chi}(L)+\widetilde{\zeta} f_{3}(L) \bar{\chi}(L) .
\end{aligned}
$$

We can rewrite 2.21 as

$$
-\left(L_{\lambda} U, V\right)_{H_{R}^{1}}+(U, V)_{H_{R}^{1}}=l(V),
$$

where

$$
H_{R}^{1}(0, L)=\left\{(u, v) \in H_{*}^{1}\left(0, l_{0}\right) \times H^{1}\left(l_{0}, L\right) \backslash u\left(l_{0}\right)=v\left(l_{0}\right)\right\}
$$

with the inner product defined by

$$
\begin{aligned}
(U, V)_{H_{R}^{1}} & =\frac{\tau_{1}}{\rho_{1}} \int_{0}^{l_{0}} u_{x} \bar{w}_{x} d x+\frac{\tau_{2}}{\rho_{2}} \int_{l_{0}}^{L} v_{x} \bar{\chi}_{x} d x-i \widetilde{\zeta} \lambda v(L) \bar{\chi}(L), \\
\left(L_{\lambda} U, V\right)_{H_{R}^{1}} & =\int_{0}^{l_{0}} \lambda^{2} u \bar{w} d x+\int_{l_{0}}^{L} \lambda^{2} v \bar{\chi} d x .
\end{aligned}
$$

Using the compactness embedding from $\left(L^{2}\left(0, l_{0}\right) \times L^{2}\left(l_{0}, L\right)\right)$ into $\left(H_{R}^{1}(0, L)\right)^{\prime}$ and from $H_{R}^{1}(0, L)$ into $L^{2}\left(0, l_{0}\right) \times L^{2}\left(l_{0}, L\right)$ we deduce that the operator $L_{\lambda}$ is compact from $L^{2}\left(0, l_{0}\right) \times L^{2}\left(l_{0}, L\right)$ into $L^{2}\left(0, l_{0}\right) \times L^{2}\left(l_{0}, L\right)$. Consequently, by Fredholm alternative, proving the existence of $U$ solution of 2.22 reduces to proving that 1 is not an eigenvalue of $L_{\lambda}$. Indeed if 1 is an eigenvalue, then there exists $U \neq 0$, such that

$$
\left(L_{\lambda} U, V\right)_{H_{R}^{1}}=(U, V)_{H_{R}^{1}}, \quad \forall V \in H_{R}^{1} .
$$

In particular for $V=U$, it follows that

$$
\lambda^{2}\left[\|u\|_{L^{2}\left(0, l_{0}\right)}^{2}+\|v\|_{L^{2}\left(l_{0}, L\right)}^{2}\right]-i \lambda \widetilde{\zeta}|v(L)|^{2}=\left\|u_{x}\right\|_{L^{2}\left(0, l_{0}\right)}^{2}+\left\|v_{x}\right\|_{L^{2}\left(l_{0}, L\right)}^{2} .
$$


Hence, we have $v(L)=0$. From (2.23), we obtain $v_{x}(L)=0$ and

$$
-\lambda^{2} u-r_{1} u_{x x}=0, \quad-\lambda^{2} v-r_{2} v_{x x}=0 .
$$

The general solutions for (2.24) are of the form

$$
u(x)=c_{1} \cos \frac{\lambda}{\sqrt{r_{1}}} x+c_{2} \sin \frac{\lambda}{\sqrt{r_{1}}} x, \quad v(x)=c_{3} \cos \frac{\lambda}{\sqrt{r_{2}}} x+c_{4} \sin \frac{\lambda}{\sqrt{r_{2}}} x .
$$

Taking into account the boundary conditions $u(0)=0$ and $v(L)=v_{x}(L)=0$, we get

$$
c_{1}=c_{3}=c_{4}=0
$$

Moreover, taking into account the boundary transmission conditions $u\left(l_{0}\right)=v\left(l_{0}\right)$ and $r_{1} u_{x}\left(l_{0}\right)=r_{2} v_{x}\left(l_{0}\right)$ we deduce that $c_{2}=0$. Then $U=0$.

Hence $i \lambda-\mathcal{A}$ is surjective for all $\lambda \in \mathbb{R}^{*}$.

Case $2: \lambda=0$ and $\eta \neq 0$. The system 2.19 is reduced to the following

$$
\begin{gathered}
-\widetilde{u}=f_{1}, \\
-\frac{\tau_{1}}{\rho_{1}} u_{x x}=f_{2}, \\
-\widetilde{v}=f_{3}, \\
-\frac{\tau_{2}}{\rho_{2}} v_{x x}=f_{4}, \\
\left(\xi^{2}+\eta\right) \phi-\widetilde{v}(L) \mu(\xi)=f_{5} .
\end{gathered}
$$

With $2.25 \mathrm{~b}$ and $2.25 \mathrm{c}$, we get

$$
u(x)=-\frac{1}{r_{1}} \int_{0}^{x} \int_{0}^{s} f_{2}(r) d r d s+C x, \quad v(x)=-\frac{1}{r_{2}} \int_{l_{0}}^{x} \int_{l_{0}}^{s} f_{4}(r) d r d s+C^{\prime} x+C^{\prime \prime} .
$$

From 2.25c and 2.25d, we have

$$
-\gamma \eta^{\alpha-1} f_{3}(L)+r_{2} v_{x}(L)+\zeta \int_{-\infty}^{+\infty} \frac{\mu(\xi) f_{5}(\xi)}{\xi^{2}+\eta} d \xi=0 .
$$

We find

$$
C^{\prime}=\frac{1}{r_{2}}\left[\int_{l_{0}}^{L} f_{4}(r) d r+\gamma \eta^{\alpha-1} f_{3}(L)-\zeta \int_{-\infty}^{+\infty} \frac{\mu(\xi) f_{5}(\xi)}{\xi^{2}+\eta} d \xi\right] .
$$

From boundary transmission conditions, we find

$$
\begin{gathered}
u\left(l_{0}\right)=v\left(l_{0}\right) \quad \Longrightarrow \quad l_{0} C-C^{\prime \prime}=\frac{1}{r_{1}} \int_{0}^{l_{0}} \int_{0}^{s} f_{2}(r) d r d s+C^{\prime} l_{0} \\
r_{1} u_{x}\left(l_{0}\right)=r_{2} v_{x}\left(l_{0}\right) \quad \Longrightarrow C r_{1}=\int_{0}^{l_{0}} \int_{0}^{s} f_{2}(r) d r+C^{\prime} r_{2} .
\end{gathered}
$$


We find

$$
C=\frac{1}{r_{1}}\left[\int_{0}^{l_{0}} \int_{0}^{s} f_{2}(r) d r+C^{\prime} r_{2}\right], \quad C^{\prime \prime}=l_{0}\left(C-C^{\prime}\right)-\frac{1}{r_{1}} \int_{0}^{l_{0}} \int_{0}^{s} f_{2}(r) d r .
$$

Hence $\mathcal{A}$ is surjective. The proof is thus complete.

Proof of Theorem 2.5. By Lemma 2.6, the operator $\mathcal{A}$ has no pure imaginary eigenvalues and by Lemma $2.7 R(i \lambda-\mathcal{A})=\mathcal{H}$ for all $\lambda \in \mathbb{R}^{*}$ and $R(i \lambda-\mathcal{A})=\mathcal{H}$ for $\lambda=0$ and for all $\eta>0$. Therefore, the closed graph theorem of Banach implies that $\sigma(\mathcal{A}) \cap i \mathbb{R}=\emptyset$ if $\eta>0$ and $\sigma(\mathcal{A}) \cap i \mathbb{R}=\{0\}$ if $\eta=0$.

\section{Lack of exponential stability}

Our goal in this section is to show that system (1.1) is not exponentially stable. We need the following well known theorem.

Theorem 3.1. (see 8 16]) Let $S(t)=e^{\mathcal{A} t}$ be a $C_{0}$-semigroup of contractions on Hilbert space. Then $S(t)$ is exponentially stable if and only if

$$
\rho(\mathcal{A}) \supseteq\{i \beta: \beta \in \mathbb{R}\} \equiv i \mathbb{R} \quad \text { and } \quad \overline{\lim }_{|\beta| \rightarrow \infty}\left\|(i \beta I-\mathcal{A})^{-1}\right\|_{\mathcal{L}(\mathcal{H})}<\infty .
$$

Our main result is

Theorem 3.2. The semigroup generated by the operator $\mathcal{A}$ is not exponentially stable.

Proof. We will examine two cases.

Case 1: $\eta=0$. We shall show that $i \lambda=0$ is not in the resolvent set of the operator $\mathcal{A}$. Indeed, noting that $(-x \sin x, 0,-x \sin x, 0,0)^{T} \in \mathcal{H}$, and denoting by $(u, \widetilde{u}, v, \widetilde{u}, \phi)^{T}$ the image of $(-x \sin x, 0,-x \sin x, 0,0)^{T}$ by $\mathcal{A}^{-1}$, we see that $\phi(\xi)=|\xi|^{(2 \alpha-5) / 2} L \sin L$. But $\phi \notin L^{2}(-\infty,+\infty)$, since $\left.\alpha \in\right] 0,1\left[\right.$ and so $(u, \widetilde{u}, v, \widetilde{u}, \phi)^{T} \notin D(\mathcal{A})$.

Case 2: $\eta \neq 0$. We aim to show that an infinite number of eigenvalues of $\mathcal{A}$ approach the imaginary axis which prevents the wave system (1.1) from being exponentially stable. Indeed we first compute the characteristic equation that gives the eigenvalues of $\mathcal{A}$. Let $\lambda$ be an eigenvalue of $\mathcal{A}$ with associated eigenvector $U=(u, \widetilde{u}, v, \widetilde{v}, \phi)^{T}$. Then $\mathcal{A} U=\lambda U$ is equivalent to

$$
\begin{gathered}
\lambda u-\widetilde{u}=0, \\
\lambda \widetilde{u}-\frac{\tau_{1}}{\rho_{1}} u_{x x}=0, \\
\lambda v-\widetilde{v}=0, \\
\lambda \widetilde{v}-\frac{\tau_{2}}{\rho_{2}} v_{x x}=0, \\
\lambda \phi+\left(\xi^{2}+\eta\right) \phi-\widetilde{v}(L) \mu(\xi)=0 .
\end{gathered}
$$


Inserting (3.1a), 3.1c) into 3.1b), 3.1d) and (3.1e), we get

$$
\begin{aligned}
\lambda^{2} u-\frac{\tau_{1}}{\rho_{1}} u_{x x}=0 & \text { in }\left(0, l_{0}\right), \\
\lambda^{2} v-\frac{\tau_{2}}{\rho_{2}} v_{x x}=0 & \text { in }\left(l_{0}, L\right), \\
\left(\lambda+\xi^{2}+\eta\right) \phi-\lambda v(L) \mu(\xi) & =0 .
\end{aligned}
$$

Using the third equation in $(3.2)$, Lemma 2.2 and the boundary conditions, we have

$$
\frac{\tau_{2}}{\rho_{2}} v_{x}(L)+\gamma \lambda(\lambda+\eta)^{\alpha-1} v(L)=0 .
$$

Finally, using the fact $u(0)=0, u\left(l_{0}\right)=v\left(l_{0}\right), \tau_{1} \rho_{2} u_{x}\left(l_{0}\right)=\tau_{2} \rho_{1} v_{x}\left(l_{0}\right)$ and $(3.3)$ we get the following system

$$
\begin{gathered}
\lambda^{2} u-\frac{\tau_{1}}{\rho_{1}} u_{x x}=0 \quad \text { in }\left(0, l_{0}\right), \\
\lambda^{2} v-\frac{\tau_{2}}{\rho_{2}} v_{x x}=0 \quad \text { in }\left(l_{0}, L\right), \\
u(0)=0, \quad u\left(l_{0}\right)=v\left(l_{0}\right), \quad \tau_{1} \rho_{2} u_{x}\left(l_{0}\right)=\tau_{2} \rho_{1} v_{x}\left(l_{0}\right), \\
\frac{\tau_{2}}{\rho_{2}} v_{x}(L)+\gamma \lambda(\lambda+\eta)^{\alpha-1} v(L)=0 .
\end{gathered}
$$

The general solutions of equations $3.4 \mathrm{a}$ and $3.4 \mathrm{~b}$ ) are given by

$$
u(x)=\sum_{i=1}^{2} c_{i} e^{t_{i} x}, \quad v(x)=\sum_{i=3}^{4} c_{i} e^{t_{i} x},
$$

where $t_{1}=\sqrt{\rho_{1} / \tau_{1}} \lambda, t_{2}=-t_{1}, t_{3}=\sqrt{\rho_{2} / \tau_{2}} \lambda, t_{4}=-t_{3}$.

Thus the boundary conditions may be written as the following system

$$
M(\lambda) C(\lambda)=\left(\begin{array}{cccc}
1 & 1 & 0 & 0 \\
e^{t_{1} l_{0}} & e^{-t_{1} l_{0}} & -e^{t_{3} l_{0}} & -e^{-t_{3} l_{0}} \\
\frac{\tau_{1}}{\rho_{1}} t_{1} e^{t_{1} l_{0}} & -\frac{\tau_{1}}{\rho_{1}} t_{1} e^{-t_{1} l_{0}} & -\frac{\tau_{2}}{\rho_{2}} t_{3} e^{t_{3} l_{0}} & \frac{\tau_{2}}{\rho_{2}} t_{3} e^{-t_{3} l_{0}} \\
0 & 0 & h\left(t_{3}\right) e^{t_{3} L} & h\left(-t_{3}\right) e^{-t_{3} L}
\end{array}\right)\left(\begin{array}{l}
c_{1} \\
c_{2} \\
c_{3} \\
c_{4}
\end{array}\right)=\left(\begin{array}{l}
0 \\
0 \\
0 \\
0
\end{array}\right),
$$

where

$$
h(r)=\frac{\tau_{2}}{\rho_{2}} r+\gamma \lambda(\lambda+\eta)^{\alpha-1} .
$$

Hence a non-trivial solution $\varphi$ exists if and only if the determinant of $M(\lambda)$ vanishes. Set $f(\lambda)=\operatorname{det} M(\lambda)$, thus the characteristic equation is $f(\lambda)=0$.

Our purpose in the sequel is to prove, thanks to Rouché's theorem, that there is a subsequence of eigenvalues for which their real part tends to 0 . 
In the sequel, since $\mathcal{A}$ is dissipative, we study the asymptotic behavior of the large eigenvalues $\lambda$ of $\mathcal{A}$ in the strip $-\alpha_{0} \leq \Re(\lambda) \leq 0$, for some $\alpha_{0}>0$ large enough and for such $\lambda$, we remark that $e^{t_{i}}, i=1,2$ remains bounded.

Case 1: $\frac{\tau_{1}}{\rho_{1}}=\frac{\tau_{2}}{\rho_{2}}$.

Lemma 3.3. There exists $N \in \mathbb{N}$ such that

$$
\left\{\lambda_{k}\right\}_{k \in \mathbb{Z}^{*},|k| \geq N} \subset \sigma(\mathcal{A})
$$

where

$$
\begin{gathered}
\lambda_{k}=i \frac{1}{r L}\left(k+\frac{1}{2}\right) \pi+\frac{\widetilde{\alpha}}{k^{1-\alpha}}+\frac{\beta}{|k|^{1-\alpha}}+o\left(\frac{1}{k^{3-\alpha}}\right), \\
k \geq N, \widetilde{\alpha} \in i \mathbb{R}, \beta \in \mathbb{R}, \beta<0, r=\sqrt{\frac{\rho_{1}}{\tau_{1}}}
\end{gathered}
$$

and $\lambda_{k}=\overline{\lambda_{-k}}$ if $k \leq-N$. Moreover for all $|k| \geq N$, the eigenvalues $\lambda_{k}$ are simple.

Proof. We divide the proof into three steps:

Step 1.

$$
\begin{aligned}
f(\lambda) & =-2 t_{1}^{2} r_{1}\left(e^{t_{1} L}+e^{-t_{1} L}\right)-2 \gamma t_{1} \lambda(\lambda+\eta)^{\alpha-1}\left(e^{t_{1} L}-e^{-t_{1} L}\right) \\
& =-2 t_{1}^{2} r_{1}\left(\left(e^{t_{1} L}+e^{-t_{1} L}\right)+\frac{\gamma}{\sqrt{\tau_{1} / \rho_{1}}} \frac{e^{t_{1} L}-e^{-t_{1} L}}{\lambda^{1-\alpha}}+o\left(\frac{1}{\lambda^{1-\alpha}}\right)\right) \\
& =-2 r_{1} t_{1}^{2} e^{-t_{1} L}\left(\left(e^{2 t_{1} L}+1\right)+\frac{\gamma}{\sqrt{\tau_{1} / \rho_{1}}} \frac{e^{2 t_{1} L}-1}{\lambda^{1-\alpha}}+o\left(\frac{1}{\lambda^{1-\alpha}}\right)\right) .
\end{aligned}
$$

We set

$$
\begin{aligned}
\tilde{f}(\lambda) & =\left(e^{2 t_{1} L}+1\right)+\frac{\gamma}{\sqrt{\tau_{1} / \rho_{1}}} \frac{e^{2 t_{1} L}-1}{\lambda^{1-\alpha}}+o\left(\frac{1}{\lambda^{1-\alpha}}\right) \\
& =f_{0}(\lambda)+\frac{f_{1}(\lambda)}{\lambda^{1-\alpha}}+o\left(\frac{1}{\lambda^{1-\alpha}}\right)
\end{aligned}
$$

where

$$
f_{0}(\lambda)=e^{2 t_{1} L}+1
$$

and

$$
f_{1}(\lambda)=\frac{\gamma}{\sqrt{\tau_{1} / \rho_{1}}}\left(e^{t_{1} L}-1\right)
$$

Note that $f_{0}$ and $f_{1}$ remain bounded in the strip $-\alpha_{0} \leq \mathcal{R}(\lambda) \leq 0$.

Step 2. We look at the roots of $f_{0}$. From (3.6), $f_{0}$ has one familie of roots that we denote $\lambda_{k}^{0}$.

$$
f_{0}(\lambda)=0 \quad \Longleftrightarrow \quad e^{2 \sqrt{\rho_{1} / \tau_{1}} \lambda L}=-1
$$


Hence

$$
2 r \lambda L=i(2 k+1) \pi, \quad k \in \mathbb{Z}, r=\sqrt{\rho_{1} / \tau_{1}},
$$

i.e.,

$$
\lambda_{k}^{0}=\frac{i(2 k+1) \pi}{2 r L}, \quad k \in \mathbb{Z} .
$$

Now with the help of Rouché's theorem, we will show that the roots of $\tilde{f}$ are close to those of $f_{0}$. Changing in (3.5) the unknown $\lambda$ by $u=2 \sqrt{\rho_{1} / \tau_{1}} \lambda L$ then 3.5 becomes

$$
\widetilde{f}(u)=\left(e^{u}+1\right)+O\left(\frac{1}{u^{1-\alpha}}\right)=f_{0}(u)+O\left(\frac{1}{u^{1-\alpha}}\right) .
$$

The roots of $f_{0}$ are $u_{k}=i\left(k+\frac{1}{2}\right) \pi /(r L), k \in \mathbb{Z}$, and setting $u=u_{k}+r e^{i t}, t \in[0,2 \pi]$, we can easily check that there exists a constant $C>0$ independent of $k$ such that $\left|e^{u}+1\right| \geq C r$ for $r$ small enough. This allows to apply Rouché's theorem. Consequently, there exists a subsequence of roots of $\tilde{f}$ which tends to the roots $u_{k}$ of $f_{0}$. Equivalently, it means that there exists $N \in \mathbb{N}$ and a subsequence $\left\{\lambda_{k}\right\}_{|k| \geq N}$ of roots of $f(\lambda)$, such that $\lambda_{k}=\lambda_{k}^{0}+o(1)$ which tends to the roots $i\left(k+\frac{1}{2}\right) \pi /(r L)$ of $f_{0}$. Finally for $|k| \geq N, \lambda_{k}$ is simple since $\lambda_{k}^{0}$ is.

Step 3. From Step 2, we can write

$$
\lambda_{k}=i \frac{1}{r L}\left(k+\frac{1}{2}\right) \pi+\varepsilon_{k} .
$$

Using (3.7), we get

$$
e^{2 r \lambda_{k} L}=-1-2 r L \varepsilon_{k}-2 r L^{2} \varepsilon_{k}^{2}+o\left(\varepsilon_{k}^{2}\right)
$$

Substituting (3.8) into (3.5), using the fact that $\tilde{f}\left(\lambda_{k}\right)=0$, we get

$$
\tilde{f}\left(\lambda_{k}\right)=-2 r L \varepsilon_{k}-\frac{2 \gamma}{\sqrt{\tau_{1} / \rho_{1}}} \frac{1}{\left(\frac{i(2 k+1) \pi}{2 r L}\right)^{1-\alpha}}+o\left(\varepsilon_{k}\right)=0,
$$

and hence

$$
\begin{aligned}
\varepsilon_{k} & =-\frac{\gamma r^{1-\alpha}}{L^{\alpha}\left(\left(k+\frac{1}{2}\right) i \pi\right)^{1-\alpha}}+o\left(\frac{1}{k^{1-\alpha}}\right) \\
& =-\frac{\gamma r^{1-\alpha}}{L^{\alpha}\left(\left(k+\frac{1}{2}\right) \pi\right)^{1-\alpha}}\left(\cos (1-\alpha) \frac{\pi}{2}-i \sin (1-\alpha) \frac{\pi}{2}\right)+o\left(\frac{1}{k^{1-\alpha}}\right) \quad \text { for } k \succeq 0 .
\end{aligned}
$$

From $(3.9)$ we have in that case $|k|^{1-\alpha} \mathcal{R} \lambda_{k} \sim \beta$, with

$$
\beta=-\frac{\gamma r^{1-\alpha}}{L^{\alpha} \pi^{1-\alpha}} \cos (1-\alpha) \frac{\pi}{2}
$$

Case 2: $\frac{\tau_{1}}{\rho_{1}} \neq \frac{\tau_{2}}{\rho_{2}}$. 
Lemma 3.4. There exists $N \in \mathbb{N}$ such that

$$
\left\{\lambda_{k}\right\}_{k \in \mathbb{Z}^{*},|k| \geq N} \subset \sigma(\mathcal{A}),
$$

where

$$
\begin{gathered}
\lambda_{k}=i \mu_{k}+\frac{\widetilde{\alpha}}{k^{1-\alpha}}+\frac{\beta}{|k|^{1-\alpha}}+o\left(\frac{1}{k^{3-\alpha}}\right), \quad k \geq N, \widetilde{\alpha} \in i \mathbb{R}, \beta \in \mathbb{R}, \beta<0, \\
\lambda_{k}=\overline{\lambda_{-k}} \quad \text { if } k \leq-N .
\end{gathered}
$$

Moreover for all $|k| \geq N$, the eigenvalues $\lambda_{k}$ are simple.

\section{Proof.}

$$
\begin{aligned}
f(\lambda)= & r_{2}^{2} t_{3}^{2}\left(e^{\left(-t_{1} l+t_{3} l-t_{3} L\right)}-e^{\left(-t_{1} l-t_{3} l+t_{3} L\right)}-e^{\left(t_{1} l+t_{3} l-t_{3} L\right)}+e^{\left(t_{1} l-t_{3} l+t_{3} L\right)}\right) \\
& -r_{2} t_{3} d\left(e^{\left(-t_{1} l+t_{3} l-t_{3} L\right)}+e^{\left(-t_{1} l-t_{3} l+t_{3} L\right)}-e^{\left(t_{1} l+t_{3} l-t_{3} L\right)}-e^{\left(t_{1} l-t_{3} l+t_{3} L\right)}\right) \\
& -r_{1} t_{1} d\left(e^{\left(-t_{1} l+t_{3} l-t_{3} L\right)}-e^{\left(-t_{1} l-t_{3} l+t_{3} L\right)}-e^{\left(t_{1} l-t_{3} l+t_{3} L\right)}+e^{\left(t_{1} l+t_{3} l-t_{3} L\right)}\right) \\
& +r_{1} r_{2} t_{1} t_{3}\left(e^{\left(-t_{1} l+t_{3} l-t_{3} L\right)}+e^{\left(-t_{1} l-t_{3} l+t_{3} L\right)}+e^{\left(t_{1} l+t_{3} l-t_{3} L\right)}+e^{\left(t_{1} l-t_{3} l+t_{3} L\right)}\right) \\
= & \sqrt{r_{2}} \lambda^{2}\left[\sqrt{r_{2}}\left(e^{t_{1} l}-e^{-t_{1} l}\right)\left(e^{(L-l) t_{3}}-e^{-(L-l) t_{3}}\right)+\sqrt{r_{1}}\left(e^{t_{1} l}+e^{-t_{1} l}\right)\left(e^{(L-l) t_{3}}+e^{-(L-l) t_{3}}\right)\right. \\
& \left.+\gamma \frac{\left(e^{t_{1} l}-e^{-t_{1} l}\right)\left(e^{(L-l) t_{3}}+e^{-(L-l) t_{3}}\right)+\frac{\sqrt{r_{1}}}{\sqrt{r_{1}}}\left(e^{t_{1} l}+e^{-t_{1} l}\right)\left(e^{(L-l) t_{3}}-e^{-(L-l) t_{3}}\right)}{\lambda^{\alpha-1}}\right] \\
= & \sqrt{r_{2}} \lambda^{2}\left[\sqrt{r_{2}}\left(e^{t_{1} l}-e^{-t_{1} l}\right)\left(e^{(L-l) t_{3}}-e^{-(L-l) t_{3}}\right)+\sqrt{r_{1}}\left(e^{t_{1} l}+e^{-t_{1} l}\right)\left(e^{(L-l) t_{3}}+e^{-(L-l) t_{3}}\right)\right. \\
& +\gamma \frac{\left(e^{t_{1} l}-e^{-t_{1} l}\right)\left(e^{(L-l) t_{3}}+e^{-(L-l) t_{3}}\right)+\frac{\sqrt{r_{1}}}{\sqrt{r_{1}}}\left(e^{t_{1} l}+e^{-t_{1} l}\right)\left(e^{(L-l) t_{3}}-e^{-(L-l) t_{3}}\right)}{} \\
& \left.+o\left(\frac{1}{\lambda^{1-\alpha}}\right)\right] .
\end{aligned}
$$

We set

$$
\begin{aligned}
\widetilde{f}(\lambda)= & \sqrt{r_{2}}\left(e^{t_{1} l}-e^{-t_{1} l}\right)\left(e^{(L-l) t_{3}}-e^{-(L-l) t_{3}}\right)+\sqrt{r_{1}}\left(e^{t_{1} l}+e^{-t_{1} l}\right)\left(e^{(L-l) t_{3}}+e^{-(L-l) t_{3}}\right) \\
& +\gamma \frac{\left(e^{t_{1} l}-e^{-t_{1} l}\right)\left(e^{(L-l) t_{3}}+e^{-(L-l) t_{3}}\right)+\frac{\sqrt{r_{1}}}{\sqrt{r_{1}}}\left(e^{t_{1} l}+e^{-t_{1} l}\right)\left(e^{(L-l) t_{3}}-e^{-(L-l) t_{3}}\right)}{\lambda^{\alpha-1}} \\
& +o\left(\frac{1}{\lambda^{1-\alpha}}\right) \\
= & f_{0}(\lambda)+\frac{f_{1}(\lambda)}{\lambda^{1-\alpha}}+o\left(\frac{1}{\lambda^{1-\alpha}}\right),
\end{aligned}
$$

where

$$
\begin{aligned}
& f_{0}(\lambda)=\sqrt{r_{2}}\left(e^{t_{1} l}-e^{-t_{1} l}\right)\left(e^{(L-l) t_{3}}-e^{-(L-l) t_{3}}\right)+\sqrt{r_{1}}\left(e^{t_{1} l}+e^{-t_{1} l}\right)\left(e^{(L-l) t_{3}}+e^{-(L-l) t_{3}}\right), \\
& f_{1}(\lambda)=\gamma\left(\left(e^{t_{1} l}-e^{-t_{1} l}\right)\left(e^{(L-l) t_{3}}+e^{-(L-l) t_{3}}\right)+\frac{\sqrt{r_{1}}}{\sqrt{r_{1}}}\left(e^{t_{1} l}+e^{-t_{1} l}\right)\left(e^{(L-l) t_{3}}-e^{-(L-l) t_{3}}\right)\right) .
\end{aligned}
$$


We look at the roots of $f_{0}$. From (3.10, $f_{0}$ has one familie of roots that we denote $\lambda_{k}^{0}$. Indeed, $f_{0}(\lambda)=0$ corresponds to the eigenvalues problem to the conservative problem associated with (2.1):

$$
\begin{array}{ll}
\rho_{1} u_{t t}(x, t)-\tau_{1} u_{x x}(x, t)=0 & \text { in }\left(0, l_{0}\right) \times(0,+\infty), \\
\rho_{2} v_{t t}(x, t)-\tau_{2} v_{x x}(x, t)=0 & \text { in }\left(l_{0}, L\right) \times(0,+\infty), \\
u\left(l_{0}, t\right)=v\left(l_{0}, t\right), \rho_{2} \tau_{1} u_{x}\left(l_{0}, t\right)=\rho_{1} \tau_{2} v_{x}\left(l_{0}, t\right) & \text { on }(0,+\infty), \\
u(0, t)=0 & \text { on }(0,+\infty), \\
v_{x}(L, t)=0 & \text { on }(0,+\infty), \\
u(x, 0)=u_{0}(x), u_{t}(x, 0)=u_{1}(x) & \text { on }\left(0, l_{0}\right), \\
v(x, 0)=v_{0}(x), v_{t}(x, 0)=v_{1}(x) & \text { on }\left(l_{0}, L\right) .
\end{array}
$$

The abstract formulation of 3.11 is

$$
\mathcal{A}_{0}\left(\begin{array}{c}
u \\
\widetilde{u} \\
v \\
\widetilde{v}
\end{array}\right)=\left(\begin{array}{c}
\widetilde{u} \\
\frac{\tau_{1}}{\rho_{1}} u_{x x} \\
\widetilde{v} \\
\frac{\tau_{2}}{\rho_{2}} v_{x x}
\end{array}\right) .
$$

The domain of $\mathcal{A}_{0}$ is

$$
D\left(\mathcal{A}_{0}\right)=\left\{\begin{array}{c}
(u, \widetilde{u}, v, \widetilde{v})^{T} \text { in } \mathcal{H}_{0}: u \in H^{2}(0, L) \cap H_{*}^{1}\left(0, l_{0}\right), \widetilde{u} \in H_{*}^{1}\left(0, l_{0}\right), \\
v \in H^{2}\left(l_{0}, L\right), \widetilde{v} \in H^{1}\left(l_{0}, L\right), u\left(l_{0}\right)=v\left(l_{0}\right), \rho_{2} \tau_{1} u_{x}\left(l_{0}\right)=\rho_{1} \tau_{2} v_{x}\left(l_{0}\right), \\
\widetilde{u}\left(l_{0}\right)=\widetilde{v}\left(l_{0}\right), v_{x}(L)=0,
\end{array}\right\}
$$

where

$$
\mathcal{H}_{0}=\left\{H_{*}^{1}\left(0, l_{0}\right) \times L^{2}\left(0, l_{0}\right) \times H^{1}\left(l_{0}, L\right) \times L^{2}\left(l_{0}, L\right) \backslash u\left(l_{0}\right)=v\left(l_{0}\right)\right\} .
$$

$\mathcal{A}_{0}$ is clearly a skew adjoint operator with a compact resolvent, then there is an orthonormal system of eigenvectors of $\mathcal{A}_{0}$ which is complete in $\mathcal{H}_{0}$. All eigenvalues of $\mathcal{A}_{0}$ are of the form $i \mu_{k}, \mu_{k} \in \mathbb{R}$. Now

$$
\begin{aligned}
f_{0}\left(i \mu_{k}\right)=0 & \Longleftrightarrow \tan \left(\sqrt{\rho_{1} / \tau_{1}} l \mu_{k}\right) \tan \left(\sqrt{\rho_{2} / \tau_{2}}(L-l) \mu_{k}\right)=\frac{\rho_{1} / \tau_{1}}{\rho_{2} / \tau_{2}} \\
& \Longleftrightarrow \tan \left(\sqrt{\rho_{1} / \tau_{1}} l \mu_{k}\right)=\frac{\rho_{1} / \tau_{1}}{\rho_{2} / \tau_{2}} \cot \left(\sqrt{\rho_{2} / \tau_{2}}(L-l) \mu_{k}\right) .
\end{aligned}
$$

By representation of graph of the functions tan and cot, we easily have $\mu_{k} \sim c k$ for large $k$ and a constant $c$ depending on parameters $\rho_{1}, \tau_{1}, \rho_{2}, \tau_{2}, l$ and $L$. Moreover, the algebraic multiplicity of $\mu_{k}$ is one. Then, we follow exactly as the case $\tau_{1} / \rho_{1}=\tau_{2} / \rho_{2}$.

The operator $\mathcal{A}$ has a non exponential decaying branche of eigenvalues. Thus the proof is complete. 


\subsection{Residual spectrum of $\mathcal{A}$}

Lemma 3.5. Let $\mathcal{A}$ be defined by (2.6). Then

$$
\mathcal{A}^{*}\left(\begin{array}{c}
u \\
\widetilde{u} \\
v \\
\widetilde{v} \\
\phi
\end{array}\right)=\left(\begin{array}{c}
-\widetilde{u} \\
-\frac{\tau_{1}}{\rho_{1}} u_{x x} \\
-\widetilde{v} \\
-\frac{\tau_{2}}{\rho_{2}} v_{x x} \\
-\left(\xi^{2}+\eta\right) \phi-\widetilde{v}(L) \mu(\xi)
\end{array}\right)
$$

with domain

$$
D\left(\mathcal{A}^{*}\right)=\left\{\begin{array}{c}
(u, \widetilde{u}, v, \widetilde{v}, \phi)^{T} \text { in } \mathcal{H}: u \in H^{2}\left(0, l_{0}\right) \cap H_{*}^{1}\left(0, l_{0}\right), \widetilde{u} \in H_{*}^{1}\left(0, l_{0}\right), \\
v \in H^{2}\left(l_{0}, L\right), \widetilde{v} \in H^{1}\left(l_{0}, L\right), u\left(l_{0}\right)=v\left(l_{0}\right), \widetilde{u}\left(l_{0}\right)=\widetilde{v}\left(l_{0}\right), \rho_{2} \tau_{1} u_{x}\left(l_{0}\right)=\rho_{1} \tau_{2} v_{x}\left(l_{0}\right), \\
-\left(\xi^{2}+\eta\right) \phi+\widetilde{v}(L) \mu(\xi) \in L^{2}(-\infty,+\infty), \\
\tau_{2} v_{x}(L)+\zeta \rho_{2} \int_{-\infty}^{+\infty} \mu(\xi) \phi(\xi, t) d \xi=0, \\
|\xi| \phi \in L^{2}(-\infty,+\infty)
\end{array}\right\} .
$$

Proof. Let $U=(u, \widetilde{u}, v, \widetilde{u}, \phi)^{T}$ and $V=\left(u_{1}, \widetilde{u}_{1}, v_{1}, \widetilde{v}_{1}, \phi_{1}\right)^{T}$. We have $\langle\mathcal{A} U, V\rangle_{\mathcal{H}}=$ $\left\langle U, \mathcal{A}^{*} V\right\rangle_{\mathcal{H}}$.

$$
\begin{aligned}
\langle\mathcal{A} U, V\rangle_{\mathcal{H}}= & \int_{0}^{l_{0}}\left(\frac{\tau_{1}}{\rho_{1}} \widetilde{u}_{x} \bar{u}_{1 x}+\frac{\tau_{1}}{\rho_{1}} \overline{\widetilde{u}}_{1} u_{x x}\right) d x+\int_{l_{0}}^{L}\left(\frac{\tau_{2}}{\rho_{2}} \widetilde{v}_{x} \bar{v}_{1 x}+\frac{\tau_{2}}{\rho_{2}} \overline{\widetilde{v}}_{1} v_{x x}\right) d x \\
& +\zeta \int_{-\infty}^{+\infty}\left[-\left(\xi^{2}+\eta\right) \phi+\widetilde{v}(L) \mu(\xi)\right] \bar{\phi}_{1} d \xi \\
= & -\int_{0}^{l_{0}}\left(\frac{\tau_{1}}{\rho_{1}} \widetilde{u}_{1 x x}+\frac{\tau_{1}}{\rho_{1}} \overline{\widetilde{u}}_{1 x} u_{x}\right) d x-\int_{l_{0}}^{L}\left(\frac{\tau_{2}}{\rho_{2}} \widetilde{v}_{1 x x}+\frac{\tau_{2}}{\rho_{2}} \overline{\widetilde{v}}_{1 x} v_{x}\right) d x \\
& +\frac{\tau_{1}}{\rho_{1}} u_{x}\left(l_{0}\right) \overline{\widetilde{u}}_{1}\left(l_{0}\right)-\frac{\tau_{2}}{\rho_{2}} v_{x}\left(l_{0}\right) \overline{\widetilde{v}}_{1}\left(l_{0}\right)+\frac{\tau_{1}}{\rho_{1}} \widetilde{u}\left(l_{0}\right) \bar{u}_{1 x}\left(l_{0}\right)-\frac{\tau_{2}}{\rho_{2}} \widetilde{v}\left(l_{0}\right) \bar{v}_{1 x}\left(l_{0}\right) \\
& +\frac{\tau_{2}}{\rho_{2}} v_{x}(L) \overline{\widetilde{v}}_{1}(L)-\zeta \int_{-\infty}^{+\infty} \phi\left[\left(\xi^{2}+\eta\right) \bar{\phi}_{1}\right] d \xi \\
& +\frac{\tau_{2}}{\rho_{2}} \widetilde{v}(L) \bar{v}_{1 x}(L)+\zeta \widetilde{v}(L) \int_{-\infty}^{+\infty} \mu(\xi) \bar{\phi}_{1} d \xi .
\end{aligned}
$$

As $\frac{\tau_{1}}{\rho_{1}} u_{x}\left(l_{0}\right)=\frac{\tau_{2}}{\rho_{2}} v_{x}\left(l_{0}\right), \widetilde{u}\left(l_{0}\right)=\widetilde{v}\left(l_{0}\right), \frac{\tau_{2}}{\rho_{2}} v_{x}(L)=-\zeta \int_{-\infty}^{+\infty} \mu(\xi) \phi d \xi$ and if we set $\frac{\tau_{1}}{\rho_{1}} u_{1 x}\left(l_{0}\right)=$ $\frac{\tau_{2}}{\rho_{2}} v_{1 x}\left(l_{0}\right)$ and $\widetilde{u}_{1}\left(l_{0}\right)=\widetilde{v}_{1}\left(l_{0}\right)$ and $\frac{\tau_{2}}{\rho_{2}} v_{1 x}(L)=-\zeta \int_{-\infty}^{+\infty} \mu(\xi) \phi_{1} d \xi$, we find

$$
\begin{aligned}
\langle\mathcal{A} U, V\rangle_{\mathcal{H}}= & -\int_{0}^{l_{0}}\left(\frac{\tau_{1}}{\rho_{1}} \widetilde{u} \bar{u}_{1 x x}+\frac{\tau_{1}}{\rho_{1}} \overline{\widetilde{u}}_{1 x} u_{x}\right) d x-\int_{l_{0}}^{L}\left(\frac{\tau_{2}}{\rho_{2}} \widetilde{v} \bar{v}_{1 x x}+\frac{\tau_{2}}{\rho_{2}} \overline{\widetilde{v}}_{1 x} v_{x}\right) d x \\
& -\zeta \int_{-\infty}^{+\infty} \phi\left[\left(\xi^{2}+\eta\right) \bar{\phi}_{1}+\mu(\xi) \overline{\widetilde{v}}_{1}(L)\right] d \xi .
\end{aligned}
$$


Theorem 3.6. $\sigma_{r}(\mathcal{A})=\emptyset$, where $\sigma_{r}(\mathcal{A})$ denotes the set of residual spectrum of $\mathcal{A}$.

Proof. Since $\lambda \in \sigma_{r}(\mathcal{A}), \bar{\lambda} \in \sigma_{p}\left(\mathcal{A}^{*}\right)$ the proof will be accomplished if we can show that $\sigma_{p}(\mathcal{A})=\sigma_{p}\left(\mathcal{A}^{*}\right)$. This is because obviously the eigenvalues of $\mathcal{A}$ are symmetric on the real axis. From 3.12 , the eigenvalue problem $\mathcal{A}^{*} Z=\lambda Z$ for $\lambda \in \mathbb{C}$ and $0 \neq Z=$ $(u, \widetilde{u}, v, \widetilde{v}, \phi) \in D\left(\mathcal{A}^{*}\right)$ we have

$$
\begin{gathered}
\lambda u+\widetilde{u}=0, \\
\lambda \widetilde{u}+\frac{\tau_{1}}{\rho_{1}} u_{x x}=0, \\
\lambda v+\widetilde{v}=0, \\
\lambda \widetilde{v}+\frac{\tau_{2}}{\rho_{2}} v_{x x}=0, \\
\lambda \phi+\left(\xi^{2}+\eta\right) \phi+\widetilde{v}(L) \mu(\xi)=0 .
\end{gathered}
$$

Inserting (3.13a), 3.13c into 3.13b), 3.13d) and (3.13e), we find

$$
\begin{aligned}
& \lambda^{2} u-\frac{\tau_{1}}{\rho_{1}} u_{x x}=0, \\
& \lambda^{2} v-\frac{\tau_{2}}{\rho_{2}} v_{x x}=0, \\
& \lambda \phi+\left(\xi^{2}+\eta\right) \phi-\lambda v(L) \mu(\xi)=0 .
\end{aligned}
$$

Using the third equation in 3.14, we easily have

$$
\gamma(\lambda+\eta)^{\alpha-1} \lambda v(L)+\frac{\tau_{2}}{\rho_{2}} \varphi_{x}(L)=0
$$

with the following conditions

$$
u(0)=0, \quad u\left(l_{0}\right)=v\left(l_{0}\right), \quad \frac{\tau_{1}}{\rho_{1}} u_{x}\left(l_{0}\right)=\frac{\tau_{2}}{\rho_{2}} v_{x}\left(l_{0}\right) .
$$

System 3.14 - 3.16 is the same as (3.4). Hence $\mathcal{A}^{*}$ has the same eigenvalues with $\mathcal{A}$. The proof is complete.

\section{Polynomial stability and optimality (for $\eta \neq 0$ )}

In the previous section, we have shown that the transmission wave system is not exponentially stable. In this section, we prove that it is polynomially stable with an optimal rate of decay when $\eta>0$. To achieve this, we use a recent result by Borichev and Tomilov [6]. Accordingly, if we consider a bounded $C_{0}$-semigroup $S(t)=e^{\mathcal{A} t}$ on a Hilbert space. If

$$
i \mathbb{R} \subset \rho(\mathcal{A}) \quad \text { and } \quad \lim _{|\beta| \rightarrow \infty} \frac{1}{\beta^{\delta}}\left\|(i \beta I-\mathcal{A})^{-1}\right\|_{\mathcal{L}(\mathcal{H})}<\infty
$$


for some $\delta>0$, then there exists $c$ such that

$$
\left\|e^{\mathcal{A} t} U_{0}\right\|^{2} \leq \frac{c}{t^{2 / \delta}}\left\|U_{0}\right\|_{D(\mathcal{A})}^{2} .
$$

Our main result is as follows.

Theorem 4.1. The semigroup $S_{\mathcal{A}}(t)_{t \geq 0}$ is polynomially stable and

$$
E(t)=\left\|S_{\mathcal{A}}(t) U_{0}\right\|_{\mathcal{H}}^{2} \leq \frac{1}{t^{2 /(1-\alpha)}}\left\|U_{0}\right\|_{D(\mathcal{A})}^{2} .
$$

Moreover, the rate of energy decay $t^{-2 /(1-\alpha)}$ is optimal for any initial data in $D(\mathcal{A})$.

Proof. We will need to study the resolvent equation $(i \lambda-\mathcal{A}) U=F$, for $\lambda \in \mathbb{R}$, namely

$$
\begin{gathered}
i \lambda u-\widetilde{u}=f_{1}, \\
i \lambda \widetilde{u}-\frac{\tau_{1}}{\rho_{1}} u_{x x}=f_{2}, \\
i \lambda v-\widetilde{v}=f_{3}, \\
i \lambda \widetilde{v}-\frac{\tau_{2}}{\rho_{2}} v_{x x}=f_{4}, \\
i \lambda \phi+\left(\xi^{2}+\eta\right) \phi-\widetilde{v}(L) \mu(\xi)=f_{5} .
\end{gathered}
$$

We divide the proof into three steps.

Step 1. Inserting (4.1a), 4.1c) into $4.1 \mathrm{~b}$ ) and $(4.1 \mathrm{~d})$, we get

$$
\begin{aligned}
\lambda^{2} u+r_{1} u_{x x} & =-\left(f_{2}+i \lambda f_{1}\right), \\
\lambda^{2} v+r_{2} v_{x x} & =-\left(f_{4}+i \lambda f_{3}\right),
\end{aligned}
$$

where $r_{1}=\tau_{1} / \rho_{1}, r_{2}=\tau_{2} / \rho_{2}$. As $u(0)=0$, then

$$
\begin{aligned}
u(x)= & c_{1} \sin \frac{\lambda}{\sqrt{r_{1}}} x-\frac{1}{\sqrt{r_{1}} \lambda} \int_{0}^{x}\left(f_{2}(\sigma)+i \lambda f_{1}(\sigma)\right) \sin \frac{\lambda}{\sqrt{r_{1}}}(x-\sigma) d \sigma, \\
v(x)= & v\left(l_{0}\right) \cos \frac{\lambda}{\sqrt{r_{2}}}\left(x-l_{0}\right)+v_{x}\left(l_{0}\right) \frac{\sqrt{r_{2}}}{\lambda} \sin \frac{\lambda}{\sqrt{r_{2}}}\left(x-l_{0}\right) \\
& -\frac{1}{\sqrt{r_{2}} \lambda} \int_{l_{0}}^{x}\left(f_{4}(\sigma)+i \lambda f_{3}(\sigma)\right) \sin \frac{\lambda}{\sqrt{r_{2}}}(x-\sigma) d \sigma
\end{aligned}
$$

and hence

$$
\begin{aligned}
u_{x}(x)= & c_{1} \frac{\lambda}{\sqrt{r_{1}}} \cos \frac{\lambda}{\sqrt{r_{1}}} x-\frac{1}{r_{1}} \int_{0}^{x}\left(f_{2}(\sigma)+i \lambda f_{1}(\sigma)\right) \cos \frac{\lambda}{\sqrt{r_{1}}}(x-\sigma) d \sigma, \\
v_{x}(x)= & -v\left(l_{0}\right) \frac{\lambda}{\sqrt{r_{2}}} \sin \frac{\lambda}{\sqrt{r_{2}}}\left(x-l_{0}\right)+v_{x}\left(l_{0}\right) \cos \frac{\lambda}{\sqrt{r_{2}}}\left(x-l_{0}\right) \\
& -\frac{1}{r_{2}} \int_{l_{0}}^{x}\left(f_{4}(\sigma)+i \lambda f_{3}(\sigma)\right) \cos \frac{\lambda}{\sqrt{r_{2}}}(x-\sigma) d \sigma .
\end{aligned}
$$


Step 2. With (4.1e), we get

$$
\phi(\xi)=\frac{\widetilde{v}(L) \mu(\xi)+f_{5}(\xi)}{i \lambda+\xi^{2}+\eta} .
$$

Inserting 4.3 in the boundary condition $\tau_{2} v_{x}(L, t)+\zeta \rho_{2} \int_{-\infty}^{+\infty} \mu(\xi) \phi(\xi, t) d \xi=0$ on $(0,+\infty)$, we deduce that

$$
r_{2} v_{x}(L)+i \gamma \lambda(i \lambda+\eta)^{\alpha-1} v(L)=\gamma(i \lambda+\eta)^{\alpha-1} f_{3}(L)-\zeta \int_{-\infty}^{+\infty} \frac{\mu(\xi) f_{5}(\xi)}{i \lambda+\xi^{2}+\eta} d \xi
$$

Then

$$
\begin{aligned}
& v\left(l_{0}\right)\left[-r_{2} \frac{\lambda}{\sqrt{r_{2}}} \sin \frac{\lambda}{\sqrt{r_{2}}}\left(L-l_{0}\right)+d \cos \frac{\lambda}{\sqrt{r_{2}}}\left(L-l_{0}\right)\right] \\
& +v_{x}\left(l_{0}\right)\left[r_{2} \cos \frac{\lambda}{\sqrt{r_{2}}}\left(L-l_{0}\right)+d \frac{\sqrt{r_{2}}}{\lambda} \sin \frac{\lambda}{\sqrt{r_{2}}}\left(L-l_{0}\right)\right] \\
& =\gamma(i \lambda+\eta)^{\alpha-1} f_{3}(L)-\zeta \int_{-\infty}^{+\infty} \frac{\mu(\xi) f_{5}(\xi)}{i \lambda+\xi^{2}+\eta} d \xi \\
& +\int_{l_{0}}^{L}\left(f_{4}(\sigma)+i \lambda f_{3}(\sigma)\right) \cos \frac{\lambda}{\sqrt{r_{2}}}(L-\sigma) d \sigma \\
& +\frac{d}{\sqrt{r_{2}} \lambda} \int_{l_{0}}^{L}\left(f_{4}(\sigma)+i \lambda f_{3}(\sigma)\right) \sin \frac{\lambda}{\sqrt{r_{2}}}(L-\sigma) d \sigma,
\end{aligned}
$$

where $d=\gamma \lambda(i \lambda+\eta)^{\alpha-1}$. Using the transmission conditions $v\left(l_{0}\right)=u\left(l_{0}\right)$ and $r_{2} v_{x}\left(l_{0}\right)=$ $r_{1} u_{x}\left(l_{0}\right)$, we get

$$
\begin{aligned}
v\left(l_{0}\right) & =c_{1} \sin \frac{\lambda}{\sqrt{r_{1}}} l_{0}-\frac{1}{\sqrt{r_{1}} \lambda} \int_{0}^{l_{0}}\left(f_{2}(\sigma)+i \lambda f_{1}(\sigma)\right) \sin \frac{\lambda}{\sqrt{r_{1}}}\left(l_{0}-\sigma\right) d \sigma, \\
v_{x}\left(l_{0}\right) & =\frac{r_{1}}{r_{2}}\left(c_{1} \frac{\lambda}{\sqrt{r_{1}}} \cos \frac{\lambda}{\sqrt{r_{1}}} l_{0}-\frac{1}{r_{1}} \int_{0}^{l_{0}}\left(f_{2}(\sigma)+i \lambda f_{1}(\sigma)\right) \cos \frac{\lambda}{\sqrt{r_{1}}}\left(l_{0}-\sigma\right) d \sigma\right) .
\end{aligned}
$$

Using (4.5), we can rewrite (4.4) as an equation in the unknown $c_{1}$ :

$$
\begin{aligned}
c_{1} & {\left[\sin \frac{\lambda}{\sqrt{r_{1}}} l_{0}\left(-\sqrt{r_{2}} \lambda \sin \frac{\lambda}{\sqrt{r_{2}}}\left(L-l_{0}\right)+d \cos \frac{\lambda}{\sqrt{r_{2}}}\left(L-l_{0}\right)\right)\right.} \\
& \left.+\cos \frac{\lambda}{\sqrt{r_{1}}} l_{0}\left(\sqrt{r_{1}} \lambda \cos \frac{\lambda}{\sqrt{r_{2}}}\left(L-l_{0}\right)+d \frac{\sqrt{r_{1}}}{\sqrt{r_{2}}} \sin \frac{\lambda}{\sqrt{r_{2}}}\left(L-l_{0}\right)\right)\right] \\
= & \gamma(i \lambda+\eta)^{\alpha-1} f_{3}(L)-\zeta \int_{-\infty}^{+\infty} \frac{\mu(\xi) f_{5}(\xi)}{i \lambda+\xi^{2}+\eta} d \xi \\
+ & \int_{l_{0}}^{L}\left(f_{4}(\sigma)+i \lambda f_{3}(\sigma)\right) \cos \frac{\lambda}{\sqrt{r_{2}}}(L-\sigma) d \sigma+\frac{d}{\sqrt{r_{2}} \lambda} \int_{l_{0}}^{L}\left(f_{4}(\sigma)+i \lambda f_{3}(\sigma)\right) \sin \frac{\lambda}{\sqrt{r_{2}}}(L-\sigma) d \sigma \\
+ & {\left[\int_{0}^{l_{0}}\left(f_{2}(\sigma)+i \lambda f_{1}(\sigma)\right) \sin \frac{\lambda}{\sqrt{r_{1}}}\left(l_{0}-\sigma\right) d \sigma\right]\left[-\frac{\sqrt{r_{2}}}{\sqrt{r_{1}}} \sin \frac{\lambda}{\sqrt{r_{2}}}\left(L-l_{0}\right)+\frac{d}{\sqrt{r_{1}} \lambda} \cos \frac{\lambda}{\sqrt{r_{2}}}\left(L-l_{0}\right)\right] } \\
+ & {\left[\int_{0}^{l_{0}}\left(f_{2}(\sigma)+i \lambda f_{1}(\sigma)\right) \cos \frac{\lambda}{\sqrt{r_{1}}}\left(l_{0}-\sigma\right) d \sigma\right]\left[\cos \frac{\lambda}{\sqrt{r_{2}}}\left(L-l_{0}\right)+\frac{d}{\sqrt{r_{2}} \lambda} \sin \frac{\lambda}{\sqrt{r_{2}}}\left(L-l_{0}\right)\right] . }
\end{aligned}
$$


Step 3. We set

$$
\begin{aligned}
g(\lambda)= & \sin \frac{\lambda}{\sqrt{r_{1}}} l_{0}\left(-\sqrt{r_{2}} \lambda \sin \frac{\lambda}{\sqrt{r_{2}}}\left(L-l_{0}\right)+d \cos \frac{\lambda}{\sqrt{r_{2}}}\left(L-l_{0}\right)\right) \\
& +\cos \frac{\lambda}{\sqrt{r_{1}}} l_{0}\left(\sqrt{r_{1}} \lambda \cos \frac{\lambda}{\sqrt{r_{2}}}\left(L-l_{0}\right)+d \frac{\sqrt{r_{1}}}{\sqrt{r_{2}}} \sin \frac{\lambda}{\sqrt{r_{2}}}\left(L-l_{0}\right)\right) \\
= & \lambda\left(\sqrt{r_{1}} \cos \frac{\lambda}{\sqrt{r_{1}}} l_{0} \cos \frac{\lambda}{\sqrt{r_{2}}}\left(L-l_{0}\right)-\sqrt{r_{2}} \sin \frac{\lambda}{\sqrt{r_{1}}} l_{0} \sin \frac{\lambda}{\sqrt{r_{2}}}\left(L-l_{0}\right)\right) \\
& +d\left(\sin \frac{\lambda}{\sqrt{r_{1}}} l_{0} \cos \frac{\lambda}{\sqrt{r_{2}}}\left(L-l_{0}\right)+\frac{\sqrt{r_{1}}}{\sqrt{r_{2}}} \cos \frac{\lambda}{\sqrt{r_{1}}} l_{0} \sin \frac{\lambda}{\sqrt{r_{2}}}\left(L-l_{0}\right)\right) .
\end{aligned}
$$

As $f_{1} \in H_{*}^{1}\left(0, l_{0}\right)$ and $f_{3} \in H^{1}\left(l_{0}, L\right)$, we have

$$
\begin{aligned}
\left|\int_{l_{0}}^{L}\left(f_{4}(\sigma)+i \lambda f_{3}(\sigma)\right) \cos \frac{\lambda}{\sqrt{r_{2}}}(L-\sigma) d \sigma\right| & \leq c\left(\left\|f_{4}\right\|_{L^{2}\left(l_{0}, L\right)}+\left\|f_{3}\right\|_{H^{1}\left(l_{0}, L\right)}\right), \\
\left|\int_{l_{0}}^{L}\left(f_{4}(\sigma)+i \lambda f_{3}(\sigma)\right) \sin \frac{\lambda}{\sqrt{r_{2}}}(L-\sigma) d \sigma\right| & \leq c\left(\left\|f_{4}\right\|_{L^{2}\left(l_{0}, L\right)}+\left\|f_{3}\right\|_{H^{1}\left(l_{0}, L\right)}\right), \\
\left|\int_{0}^{l_{0}}\left(f_{2}(\sigma)+i \lambda f_{1}(\sigma)\right) \sin \frac{\lambda}{\sqrt{r_{1}}}\left(l_{0}-\sigma\right) d \sigma\right| & \leq c\left(\left\|f_{2}\right\|_{L^{2}\left(0, l_{0}\right)}+\left\|f_{1}\right\|_{H^{1}\left(0, l_{0}\right)}\right), \\
\left|\int_{0}^{l_{0}}\left(f_{2}(\sigma)+i \lambda f_{1}(\sigma)\right) \cos \frac{\lambda}{\sqrt{r_{1}}}\left(l_{0}-\sigma\right) d \sigma\right| & \leq c\left(\left\|f_{2}\right\|_{L^{2}\left(0, l_{0}\right)}+\left\|f_{1}\right\|_{H^{1}\left(0, l_{0}\right)}\right) .
\end{aligned}
$$

If $r_{1}=r_{2}$, then

$$
g(\lambda)=\sqrt{r_{1}} \lambda \cos \frac{\lambda}{\sqrt{r_{1}}} L+d \sin \frac{\lambda}{\sqrt{r_{1}}} L .
$$

We can easily prove that

$$
|g(\lambda)| \geq c|\lambda|^{\alpha} \quad \text { for } \lambda \text { large. }
$$

Hence

$$
\left|c_{1}\right| \leq c|\lambda|^{-\alpha} \text { for } \lambda \text { large. }
$$

Then, we deduce that

$$
\left\|u_{x}\right\|_{L^{2}\left(0, l_{0}\right)} \leq c|\lambda|^{1-\alpha} \quad \text { for } \lambda \text { large. }
$$

Moreover, as $v\left(l_{0}\right)=u\left(l_{0}\right)$ and $r_{2} v_{x}\left(l_{0}\right)=r_{1} u_{x}\left(l_{0}\right)$, we have

$$
\left|v\left(l_{0}\right)\right| \leq c|\lambda|^{-\alpha}, \quad\left|v_{x}\left(l_{0}\right)\right| \leq c|\lambda|^{1-\alpha} \quad \text { for } \lambda \text { large. }
$$

Hence

$$
\left\|v_{x}\right\|_{L^{2}\left(l_{0}, L\right)} \leq c|\lambda|^{1-\alpha} \quad \text { for } \lambda \text { large. }
$$

From 4.1a), 4.1c and 4.2, we have

$$
\|\widetilde{u}\|_{L^{2}\left(0, l_{0}\right)},\|\widetilde{v}\|_{L^{2}\left(l_{0}, L\right)} \leq c|\lambda|^{1-\alpha} \quad \text { for } \lambda \text { large. }
$$


From 4.3, we get

$$
\begin{aligned}
\|\phi\|_{L^{2}(-\infty, \infty)} & \leq|\widetilde{v}(L)|\left\|\frac{\mu(\xi)}{i \lambda+\xi^{2}+\eta}\right\|_{L^{2}(-\infty, \infty)}+\left\|\frac{f_{5}(\xi)}{i \lambda+\xi^{2}+\eta}\right\|_{L^{2}(-\infty, \infty)} \\
& \leq c|\lambda|^{-\alpha / 2}\left(\left\|f_{1}\right\|_{H^{1}\left(0, l_{0}\right)}+\left\|f_{2}\right\|_{L^{2}\left(l_{0}, L\right)}\right)+c \frac{1}{|\lambda|}\left\|f_{5}\right\|_{L^{2}(-\infty, \infty)}
\end{aligned}
$$

Thus, we conclude that

$$
\left\|(i \lambda I-\mathcal{A})^{-1}\right\|_{\mathcal{H}} \leq c|\lambda|^{1-\alpha} \quad \text { as }|\lambda| \rightarrow \infty .
$$

If $r_{1} \neq r_{2}$, then from 4.6 , system

$$
\begin{gathered}
\sqrt{r_{1}} \cos \frac{\lambda}{\sqrt{r_{1}}} l_{0} \cos \frac{\lambda}{\sqrt{r_{2}}}\left(L-l_{0}\right)-\sqrt{r_{2}} \sin \frac{\lambda}{\sqrt{r_{1}}} l_{0} \sin \frac{\lambda}{\sqrt{r_{2}}}\left(L-l_{0}\right)=0, \\
\sin \frac{\lambda}{\sqrt{r_{1}}} l_{0} \cos \frac{\lambda}{\sqrt{r_{2}}}\left(L-l_{0}\right)+\frac{\sqrt{r_{1}}}{\sqrt{r_{2}}} \cos \frac{\lambda}{\sqrt{r_{1}}} l_{0} \sin \frac{\lambda}{\sqrt{r_{2}}}\left(L-l_{0}\right)=0
\end{gathered}
$$

is equivalent to

$$
\tan \frac{\lambda}{\sqrt{r_{1}}} l_{0} \tan \frac{\lambda}{\sqrt{r_{2}}}\left(L-l_{0}\right)=\sqrt{\frac{r_{1}}{r_{2}}}, \quad \frac{\tan \frac{\lambda}{\sqrt{r_{1}}} l_{0}}{\tan \frac{\lambda}{\sqrt{r_{2}}}\left(L-l_{0}\right)}=-\sqrt{\frac{r_{1}}{r_{2}}},
$$

which is impossible. Therefore, in all cases, we have

$$
|g(\lambda)| \geq c|\lambda|^{\alpha} \quad \text { for } \lambda \text { large. }
$$

Similar to the case $r_{1}=r_{2}$, we obtain the estimation 4.7.

Besides, we prove that the decay rate is optimal. Indeed, the decay rate is consistent with the asymptotic expansion of eigenvalues which shows a behavior of the real part like $k^{-(1-\alpha)}$.

\section{Acknowledgments}

The authors would like to thank very much the referees for their constructive comments and suggestions that helped to improve this article.

\section{References}

[1] Z. Achouri, N. E. Amroun and A. Benaissa, The Euler-Bernoulli beam equation with boundary dissipation of fractional derivative type, Math. Methods Appl. Sci. 40 (2017), no. 11, 3837-3854.

[2] W. Arendt and C. J. K. Batty, Tauberian theorems and stability of one-parameter semigroups, Trans. Amer. Math. Soc. 306 (1988), no. 2, 837-852. 
[3] R. L. Bagley and P. J. Torvik, A theoretical basis for the application of fractional calculus to viscoelasticity, J. Rheol. 27 (1983), no. 3, 201-210.

[4] - Fractional calculus - A different approach to the analysis of viscoelastically damped structures, AIAA J. 21 (1983), no. 5, 741-748.

[5] E. Blanc, G. Chiavassa and B. Lombard, Biot-JKD model: simulation of $1 D$ transient poroelastic waves with fractional derivatives, J. Comput. Phys. 237 (2013), 1-20.

[6] A. Borichev and Y. Tomilov, Optimal polynomial decay of functions and operator semigroups, Math. Ann. 347 (2010), no. 2, 455-478.

[7] U. J. Choi and R. C. MacCamy, Fractional order Volterra equations with applications to elasticity, J. Math. Anal. Appl. 139 (1989), no. 2, 448-464.

[8] F. L. Huang, Characteristic conditions for exponential stability of linear dynamical systems in Hilbert spaces, Ann. Differential Equations 1 (1985), no. 1, 43-55.

[9] V. Komornik, Exact Controllability and Stabilization: The multiplier method, RAM: Research in Applied Mathematics, Masson, Paris; John Wiley \& Sons, Chichester, 1994.

[10] Z.-H. Luo, B.-Z. Guo and O. Morgul, Stability and Stabilization of Infinite Dimensional Systems with Applications, Communications and Control Engineering Series, Springer-Verlag London, London, 1999.

[11] Yu. I. Lyubich and V. Q. Phóng, Asymptotic stability of linear differential equations in Banach spaces, Studia Math. 88 (1988), no. 1, 37-42.

[12] M. Mainardi and E. Bonetti, The application of real-order derivatives in linear viscoelasticity, in: Progress and Trends in Rheology II, 64-67, Steinkopff, Heidelberg, 1988.

[13] B. Mbodje, Wave energy decay under fractional derivative controls, IMA J. Math. Control Inform. 23 (2006), no. 2, 237-257.

[14] B. Mbodje and G. Montseny, Boundary fractional derivative control of the wave equation, IEEE Trans. Automat. Control 40 (1995), no. 2, 378-382.

[15] I. Podlubny, Fractional Differential Equations: An introduction to fractional derivatives, fractional differential equations, to methods of their solution and some of their applications, Mathematics in Science and Engineering 198, Academic Press, San Diego, CA, 1999. 
[16] J. Prüss, On the spectrum of $C_{0}$-semigroups, Trans. Amer. Math. Soc. 284 (1984), no. $2,847-857$.

[17] A. J. A. Ramos and M. W. P. Souza, Equivalence between observability at the boundary and stabilization for transmission problem of the wave equation, Z. Angew. Math. Phys. 68 (2017), no. 2, Art. 48, 11 pp.

[18] S. G. Samko, A. A. Kilbas and O. I. Marichev, Fractional Integrals and Derivatives: Theory and applications, Gordon and Breach Science Publishers, Yverdon, 1993.

[19] P. J. Torvik and R. L. Bagley, On the appearance of the fractional derivative in the behavior of real material, J. Appl. Mech. 51 (1984), no. 2, 294-298.

Halim Atoui and Abbes Benaissa

Laboratory of Analysis and Control of PDEs, Djillali Liabes University, P. O. Box 89, Sidi Bel Abbes 22000, Algeria

E-mail address: atouihalim@yahoo.com, benaissa_abbes@yahoo.com 